\title{
Commerce et système d'information : le cas des vendeurs de pagnes à Brazzaville (Congo)*
}

\author{
Mathias Marie A. Ndinga**
}

\section{Résumé}

L'article a pour objet l'analyse de la place et de l'incidence des Technologies de l'Information et de la Communication (TIC) dans le commerce des pagnes à Brazzaville. L'investigation est effectuée suivant trois axes : l'accès, les modalités d'utilisation et l'analyse d'impact. Il ressort des différentes analyses que le niveau d'accès aux TICs est bas lorsque l'on considère l'ensemble des vendeurs ou seulement les hommes ou les femmes ; bien que le pourcentage des hommes y soit supérieur à celui des femmes. Les TICs sont très peu utilisées surtout dans les relations entre les consommateurs et les vendeurs (grossistes et détaillants). L'utilisation des TICs pour faire circuler l'information entre les différents intervenants sur ce marché a montré des différences importantes entre les hommes disposant d'un téléphone mobile, d'une part, et entre les hommes et les femmes, d'autre part. En ce qui concerne l'incidence des TICs sur le commerce des pagnes, l'on a relevé que les proportions des vendeurs de pagnes qui perçoivent les gains dus aux TICs sont faibles. C'est toujours dans le

* Ce texte fait suite à ma participation à l'institut sur le genre organiser par le CODESRIA en 2002. Il a bénéficié d'un soutien financier du CODESRIA à qui j'adresse tous mes remerciements. Ces derniers s'adressent également aux attachés de recherche du CREP qui ont pris part à la réalisation de l'enquête. Il s’agit de Boukaka Nzongana Homère Rosian, Badila Fustel Romaric et Mbou Likibi Symphorien Gaspard. Enfin, je remercie les directeurs de l'institut sur le genre 2002 (Sheila Bunwaree et Jean-Bernard Ouédraogo) et les lecteurs anonymes pour leurs commentaires qui ont été des plus précieux. Je reste toutefois seul responsable d'éventuelles insuffisances de ce texte.

** Centre de Recherche et d’Études Prospectives, Université de Brazzaville, Congo.E-mail : ndinga01@yahoo.fr 
groupe des hommes que les résultats sont importants par rapport à celui des femmes. L'analyse économétrique a révélé que c'est l'utilisation des TICs dans les relations avec les fournisseurs qui a un impact significatif sur les recettes journalières. Enfin, les orientations de politique ont été formulées pour permettre une large diffusion des TICs et leur maîtrise par les populations en général et les vendeurs de pagnes en particulier.

\begin{abstract}
This paper seeks to establish the impact of information and communication technologies (ICTs) on the trade in wrapper cloth in Brazzaville, Congo. The study focused on access, use and impact and concluding that the level of access is low in general and for men and women taken separately, although men have more access than women. There is very little evidence of ICT use in dealings between consumer and traders (wholesalers and retailers alike). There is significant difference in ICT use between men who have mobile phones on the one hand, and between men and women on the other hand. Among traders in wrapper cloth, the proportion who perceive access to ICT as beneficial to the gains they make is low. Results are more encouraging amongst men than women, with econometric analysis showing a significant impact of ICTs on daily earnings in dealings with suppliers. Finally, the policy is to encourage widespread use of ICTs by the population in general, and by wrapper cloth traders in particular.
\end{abstract}

\title{
Introduction
}

Développées dans les pays avancés dans les années 1980, les Technologies de l'Information et de la Communication (TIC) constituent aujourd'hui l'un des principaux vecteurs de la mondialisation. Ces technologies (le numérique, l'Internet, le téléphone portable,...) ont ouvert une nouvelle ère d'interdépendance des réseaux qui bouleversent le mode de création, de diffusion et d'utilisation de la technologie.

Cette dernière qui est représentée par la transformation des conditions de la production et des échanges induits par la diffusion des Technologies de l’Information et de la Communication s’est développée progressivement en Afrique dans les années 1990. Les TICs, les plus utilisées sont : le téléphone mobile et l'Internet à petite échelle. En effet, le continent africain comptait à peine deux (2) millions d'utilisateurs de téléphones portables en 1999 et devrait compter trente (30) millions en 2001 soit près d'une fois et demi le nombre de lignes fixes.

En 2005, cent (100) millions d'abonnés de téléphones mobiles sont attendus (Marot 2001). S’agissant de l'Internet, poursuit le même auteur, l’Afrique est au début de sa révolution. On estime à 4,4 millions le nombre d'utilisateurs d'Internet sur le continent début 2001 (Afrique du Sud et pays maghrébins surtout), soit 0,5 pour cent des connections mondiales contre 50 pour cent 
pour les pays développés. Le niveau de diffusion devrait atteindre 1 pour cent seulement en 2005. Ces chiffres illustrent, pour le téléphone mobile et l'Internet, une diffusion à deux vitesses sur le continent : accélérée pour le portable et lente pour l'Internet. Ce constat est également valable pour la République du Congo (Brazzaville).

En effet, en République du Congo, à la faveur de la libéralisation du marché depuis 1997, le téléphone cellulaire s’est fortement développé. Sur les 108 400 abonnés aux différents réseaux téléphoniques, 95 pour cent le sont par le mobile (Marchés tropicaux 2001). Trois opérateurs sont présents sur le marché congolais : Cyrus, Celtel et Libertis. À ces trois opérateurs on peut ajouter l'Office national des Postes et Télécommunications (ONPT) pour le fixe. Quant à l'Internet, il est encore balbutiant avec à peine une centaine de Cybercafés dans le pays (principalement à Brazzaville et à Pointe-Noire). Jusqu’à ces derniers mois, le serveur fournisseur d'accès était Congo Net, filiale de l'ONPT dont les connections passent via l'Afrique du Sud. Il a été rejoint en juin 2001 par la société panafricaine Afripa-télécom et par Celteltélécom.

Cet article est justifié par la nécessité de vérifier si ces Technologies de l'Information et de la Communication ont les mêmes effets à Brazzaville qu'ailleurs dans le monde et surtout si elles sont réductrices ou amplificatrices des inégalités entre genre dans le commerce des pagnes. Le choix de cette dernière activité se justifie par le fait que le commerce des pagnes a la spécificité d'impliquer autant les hommes que les femmes. S'agissant des femmes, elle a donné lieu à une classe de femmes appelée « Les mamans de Lomé », du nom de la ville où elles s’approvisionnent, le Congo ne produisant plus de pagnes.

Ainsi, la problématique qui sous-tend cette réflexion sur l'incidence des TICs dans le commerce des pagnes à Brazzaville découle à la fois du contexte de mondialisation caractérisé par le développement des TICs et de l'accélération des changements qui interviennent dans les relations entre hommes et femmes dans les sociétés africaines (Locho 1996). C’est pourquoi l'investigation s'articule autour des interrogations suivantes : Qu'est ce qui détermine l'accès aux TICs des hommes et des femmes qui exercent cette activité ? Quel est le degré d'utilisation des TICs par les vendeurs de pagnes à Brazzaville ? Qu'est ce qui diffère les hommes et les femmes dans l'accès et l'utilisation des TICs dans l'exercice de leur activité ? Quel est le rôle des TICs dans le commerce des pagnes à Brazzaville?

$\mathrm{Au}$ regard de ces préoccupations, l'objectif principal poursuivi par la présente recherche est l'analyse de la place et l'incidence des TICs dans le commerce des pagnes à Brazzaville. La recherche est effectuée suivant trois 
axes. Avant d'étudier l'incidence des TICs dans le commerce des pagnes, il est indispensable de procéder à une analyse des conditions d'accès, et de procéder à une étude des modalités d'utilisation des TICs dans cette activité, car ces deux premiers axes déterminent l'incidence des TICs sur le commerce des pagnes.

L'observation de la réalité quotidienne des hommes et des femmes qui évoluent dans le commerce des pagnes à Brazzaville amène à soutenir que l'accès aux technologies de l'information et de la communication est inégal à au moins deux niveaux : vertical (hommes-femmes) et horizontal (hommeshommes et femmes-femmes). On y relève également des différences d'utilisation. Ces différences sont dues principalement au manque de formation et aux coûts des équipements. Il est aussi soutenu que les Technologies de l'Information et de la Communication, par l'accès facile à l'information qu'elles permettent, contribuent à la réduction des coûts et améliorent la qualité des services aussi bien au niveau des hommes que des femmes.

Le présent article s'articule autour de la présentation de l'approche du genre utilisée dans l'analyse et la méthodologie de la collecte des données de terrain. Ensuite sont analysées les questions relatives à l'accès aux TICs et les différentes modalités de leur utilisation. Enfin, il est fait un examen des incidences des TICs.

\section{L’approche " genre ", observation du champ d'étude et modes d'investigation}

Il est indispensable de procéder à la présentation du mode d'approche utilisé dans la présente étude ainsi que la méthodologie de collecte des données ayant servi à l'analyse. En effet, L'approche en termes de genre qui est privilégiée dans cette étude est le résultat d'une évolution dans la manière d'aborder les problèmes des femmes. L'approche "Gender and Development" succède aux approches Women in Development et Women and Development, auxquelles on reproche principalement d'impliquer que le problème de fond est constitué par les femmes alors qu'il s'agit bien plus de l'allocation des ressources entre les hommes et les femmes (Bisilliat 2000). C'est ainsi que les approches précédentes ont été remises en cause et l'utilisation de l'approche Gender and Development s'est peu à peu répandue.

L'approche Gender and Development peut être utilisée pour analyser les rapports sociaux, en intégrant leurs différences, leurs complémentarités, leurs synergies et parfois leurs conflits. Le genre permet de prendre en compte les relations de pouvoir inégales entre hommes et femmes au sein de la société et de reconnaître qu'elles sont responsables d'un partage inéquitable des ressources, des responsabilités et du pouvoir entre femmes et hommes. 
L'analyse différenciée par genre permet aussi de prendre en compte d'autres catégories de concernés et d'autres spécificités car les catégories hommes et femmes ne sont pas homogènes : tranches d'âge, éthnicité, statut dominant / dominé, niveau de richesse / pauvreté, religion, catégories socioprofessionnelles, etc.

L'analyse des différences socio-économiques et de genre des populations concernées par une intervention de développement est un préalable à une analyse complète des problèmes qui conditionnent largement la qualité d'un projet de développement. Dans le cadre de la présente étude, l'analyse en terme de genre permet d'intégrer la prise en compte des dynamiques de changements sociaux dans une situation de mondialisation marquée par le développement des TICs, et le suivi ultérieur de leur évolution, notamment au regard de la réduction ou de l'aggravation des disparités entre hommes et femmes dans le commerce des pagnes en particulier.

Cette approche peut être associée à l'approche néoclassique du marché. En effet, il y a lieu de relever que sur le plan économique l'analyse du marché a connu des développements importants depuis l'article précurseur de George Akerlof (1970) sur les problèmes posés par l'absence d'information sur le marché. Ce dernier qui constitue notre champ d'investigation est passé dans l'analyse néoclassique d'une situation de parfaite information (le tâtonnement walrasien) à une situation d'information asymétrique caractérisée par la sélection adverse et / ou l'aléa de moralité. Ainsi, l'acquisition de l'information peut conférer à un agent une position dominante par rapport à son concurrent. C'est dans cette perspective que les théories de l'information seront utilisées dans l'analyse du marché des pagnes à Brazzaville.

En ce qui concerne la collecte des données, il y a lieu de mentionner d'abord qu'à différents niveaux de cette recherche, il est fait usage aussi bien des données primaires que des données secondaires. En tenant compte de l'objet de la présente étude à savoir la place et l'incidence des TICs dans le commerce des pagnes à Brazzaville, la méthodologie suivante basée sur deux modes d'investigation a été appliquée.

La recherche documentaire qui a consisté en l'exploitation des informations diverses à partir d'une analyse du contenu de la documentation disponible sur les TICs dans le commerce en général et le commerce des pagnes en particulier. Ont donc été recensés : les documents de recherche, les articles et tout autre document en relation avec l'objet de cette recherche. Elle s'est faite au niveau des différents organismes, institutions, ministères, notamment celui en charge des questions féminines.

L'enquête de terrain, réalisée dans une perspective pluridisciplinaire et participative, a permis d'avoir des informations sur les changements intervenus dans l'approvisionnement et la vente des pagnes à la suite de la mondialisation, 
notamment le développement des TICs. L’observation préliminaire de l'environnement dans lequel se déroule le commerce des pagnes a permis la collecte d'un certain nombre d'informations sur les effectifs, les regroupements associatifs, les regroupements par bloc dans les marchés et le nombre de points de communication. ${ }^{1}$

S'agissant des effectifs, il convient d'abord de relever qu'au moment du passage des enquêteurs tous les vendeurs ne pouvaient pas être présents sur le marché. Sur les vendeurs absents, les renseignements ont été obtenus auprès des vendeurs présents. Ils portent sur leurs sexes et leurs emplacements. Malgré cet effort, Il est fort probable que des vendeurs isolés et / ou absents au moment de ce passage n'ont pas été pris en compte. Cependant cette erreur était très minime dans la mesure où en confrontant les chiffres obtenus à ceux des différents comités de marché, ceux obtenus par les enquêteurs étaient toujours en dépassement de deux à trois vendeurs.

Cette première activité qui a consisté en l'observation du champ d'étude a permis de faire le constat suivant lequel, les hommes sont minoritaires dans la vente des pagnes sur les différents marchés de Brazzaville. Il représente à peine un pourcentage de 10,71 pour cent du total des vendeurs de pagnes recensés. En considérant les marchés de manière séparée, les hommes sont absents dans la vente des pagnes dans les marchés de Bourreau, Commission et de Ouenzé. Leur participation est plus importante dans les marchés de Moungali, de Mikalou et de Bouémba où ils atteignent 20 pour cent et plus. Au regard de ce premier constat, il peut être avancé que le commerce des pagnes est une activité presque exclusivement féminine à Brazzaville.

La configuration des effectifs observés sur le marché donne la répartition suivante des 140 vendeurs de pagnes interrogés : 14 au marché de Potopoto, 8 au marché de Talangaï, 12 au marché de Bouémba, 1 aux marchés « Commission » et « Bourreau », 60 au marché de Moungali, 25 au marché de Ouenzé et 60 au marché de Total.

En ce qui concerne la disposition des vendeurs de pagnes dans les différents marchés, il a été observé également que les vendeurs de pagnes se regroupaient par bloc dans les plus grands marchés. C’est ainsi qu'au marché de Total il a été enregistré trois (3) blocs de vendeurs de pagnes composés chacun de 55, 20 et 12 vendeurs. Au marché de Ouenzé, deux principaux blocs de 18 et 24 vendeurs de pagnes ont été répertoriés et auxquels il faut ajouter un regroupement de 4 vendeurs isolés dans un coin du marché. Le marché de Poto-poto comprend quant à lui un bloc de 13 vendeurs et un regroupement de 6 vendeurs. Dans le reste des marchés, il a été observé des regroupements de vendeurs sur 2 à 6 tables de suite.

L'observation préliminaire du champ de cette étude nous a permis également d'identifier quelques organisations des vendeurs de pagnes. Au 
marché de Poto-poto, les vendeurs de pagnes sont regroupés dans une coopérative créée en 1982. Elle comprend dix-neuf membres dont deux (2) hommes et dix-sept (17) femmes. Elle est dirigée par madame Marie T. qui est vendeuse de pagnes depuis 1981.

Une autre coopérative a été identifiée au marché Total. Elle est composée de soixante (60) membres et est de ce fait la plus grande. Elle est dirigée par madame Gertrude B. Dans les autres marchés, il a été identifié des organisations qui ne sont pas aussi structurées comme dans les deux marchés déjà cités, mais dont les vendeurs de pagnes ont leur président. Son rôle se limite en général à servir de relais entre le comité de marché et les vendeurs de pagnes.

Toujours en rapport avec l'objet de cette étude, l'attention a été portée sur les points de communication dans et autour des différents marchés. Plus précisément la démarche a consisté ici à dénombrer les cybercafés et les cabines téléphoniques dans les marchés et sur les avenues et les ruelles qui délimitent ces différents marchés.

Ce travail préliminaire a permis de faire deux constats sommaires quant au niveau de pénétration des technologies de l'information et de la communication. Le premier est l'évolution à deux vitesses de l'Internet (très lente) et du téléphone mobile (très vite). Le marché « Total » qui est le plus grand de la ville compte trois cybercafés, ceux de Moungali et de Poto-poto, deux chacun, tandis que celui de Talangaï n'en possède pas. Les chiffres obtenus au niveau des cybercafés laissent penser que l'Internet n'est pas encore un phénomène de grande ampleur d'autant plus que le marché et ses abords devraient être des lieux privilégiés de leur présence en raison de l'importance des transactions. Le nombre de cabines téléphoniques est plus important (50 au marché "Total », 30 au marché de Poto-poto et 28 au marché de Moungali), mais ce qui est intéressant c'est le fait que ces cabines fonctionnent en majorité avec le téléphone mobile, qui selon certains propriétaires est plus rentable que le téléphone fixe.

Le deuxième constat est que les points de communication sont plus importants dans les grands marchés (Total, Poto-poto, Moungali, Ouenzé et Talangaï). Les marchés périphériques (Bouémba et Mikalou) sont ceux qui ont le moins de points de communication. Ce deuxième constat nous amène à avancer que le niveau d'activité tout comme le niveau de développement déterminent l'accès aux TICs. Mais au-delà de ces considérations, il faut dire que la présence même modeste de ces points de communication permet aux commerçants qui ne disposent pas de moyens personnels de communication d'accéder à ces outils. D'où l'importance d'examiner de manière plus approfondie les questions relatives à l’accès aux TICs. 


\section{L'accès des vendeurs de pagnes aux TICs}

La nécessité d'aborder ce thème par les questions liées à l'accès se trouve justifiée dans la mesure où la mondialisation, qui a pour vecteur les technologies de l'information et de la communication n'est pas un phénomène linéaire. Les inégalités qui l'accompagnent amènent certains auteurs comme Edem Kodjo (2000) à dire que dans ce nouveau monde de l'information et de la communication, placé sous le signe de la concurrence et du profit, les groupes défavorisés - que ce soit à l'intérieur de chaque société ou au niveau international - risquent de se retrouver exclus, à moins que nos États ne mettent en place des politiques claires et imaginatives afin de bénéficier des retombées de la révolution en cours. Ainsi, l'analyse des questions d'accès dans le cadre du commerce des pagnes permet d'avoir un premier éclairage sur la situation des hommes et des femmes évoluant dans cette activité. Trois points constituent la pierre angulaire de cette analyse. Le premier point est consacré à l'évaluation du degré de pénétration des TICs dans cette activité. Le second point est consacré à l'analyse des déterminants de l'accès aux TICs et le troisième est axé sur des contraintes.

\section{Le degré d'accès des vendeurs de pagnes aux TICs}

En se référant à tous les gains que les Techniques de l'Information et de la Communication sont supposées apporter au domaine des services, de manière théorique, il y a lieu de dire que le degré d'accès des vendeurs de pagnes est dans l'ensemble très faible. Les résultats de l'enquête montrent que moins de 50 pour cent des vendeurs de pagnes sont détenteurs du téléphone mobile. Autrement, un peu plus de la moitié des vendeurs de pagnes ne sont pas encore détenteurs d'un téléphone mobile. Un autre constat est que ce résultat d'ensemble ne laisse pas apparaître les disparités entre les groupes. En effet, la lecture sous cet angle permet de mettre en évidence ce que l'on peut déjà qualifier de « masculinisation $»^{2}$ du téléphone mobile dans le commerce des pagnes.

Pour mieux saisir ce phénomène de « masculinisation » du téléphone mobile dans le commerce des pagnes, un intérêt a été porté sur le mode d'acquisition du téléphone mobile. L'enquête a révélé que 81,8 pour cent des hommes ont acheté eux-mêmes leurs téléphones, alors que ce pourcentage n'est que de 46, pour cent pour les femmes. Plus encore, en s'intéressant à la provenance du téléphone offert, les résultats de l'enquête suggèrent que 56,7 pour cent des vendeurs de pagnes aient reçu des téléphones offerts par des conjoints, 31,03 pour cent par des parents et 10,34 pour cent par des amis.

Les résultats par groupe ont montré également d'importantes disparités entre les deux groupes. Les téléphones portables offerts aux hommes 
proviennent essentiellement des parents et des amis à pourcentage égal de 50 pour cent. S'agissant des femmes, les téléphones reçus proviennent essentiellement des conjoints (62,96 pour cent), suivis des parents (29,63 pour cent) et enfin des amis (7,41 pour cent). Cet ensemble de résultats apporte le premier éclairage suivant lequel l'accès au téléphone portable est beaucoup plus facile aux hommes plutôt qu'aux femmes.

Au niveau de l'Internet, il a été retenu comme indicateur le pourcentage des vendeurs possédant ou non une adresse électronique. Sur le choix de cet indicateur il y a lieu de dire que contrairement à la possession d'un ordinateur ou d'une ligne Internet personnelle qui sont difficile à acquérir, l'adresse électronique s'obtient à un prix allant de 500 à 750 F CFA 3 .

Ainsi, sans des connaissances en informatique, une personne peut naviguer via l'assistance qui lui est offerte par les maisons services. Mais malgré cette facilité, la situation est encore beaucoup plus critique tant pour les hommes que pour les femmes. Les résultats de l'enquête sur ce sujet montrent que la proportion des vendeurs de pagnes disposant d'une adresse électronique est très faible (1,4 pour cent). Lorsque l'on considère le groupe des femmes, cette proportion n'atteint pas 1 pour cent. Au niveau des hommes elle est de 6,3 pour cent. Ici encore les hommes sont les plus importants à accéder à l'Internet. L'accès au téléphone mobile comme à l'Internet est faible. Pour améliorer ce niveau d'accès, il est indispensable de connaître ses déterminants les plus significatifs au niveau individuel et au niveau global.

\section{Les déterminants de l'accès aux TICs au niveau des vendeurs de pagnes}

L’analyse précédente a montré que le taux de pénétration des TICs, notamment du téléphone mobile, n'est pas non plus élevé au regard de l'importance que l'on attribue à cette innovation dans ce genre d'activité. Dans le but d'identifier les déterminants les plus significatifs c'est-à-dire sur lesquels une action peut être menée afin d'améliorer le taux de pénétration, un modèle logistique binaire a été estimé. Il relie une variable binaire prenant la valeur 1 lorsque le vendeur de pagnes dispose d'un téléphone mobile et 0 autrement et un ensemble de variables que l'on peut classer en trois catégories. Le premier type de variables se rapporte aux aspects démographiques. Il s'agit le l'âge du vendeur et de son sexe.

Le deuxième type de variables a trait à l'éducation. Il a été retenu le niveau d'instruction du vendeur de pagne et le diplôme le plus élevé de ce dernier. Enfin le troisième type de variables se rapporte à l'activité. Il s'agit de la recette moyenne journalière et de la localisation des fournisseurs locaux ou étrangers. 
Les résultats de l'estimation du modèle permettent de faire quelques commentaires. Premièrement, la qualité de la régression est relativement bonne, la capacité de prédiction du modèle est de 76 pour cent. S'agissant du test du ratio de vraisemblance, la statistique de Khi-deux (c2) qui est de 33,787 est significative au seuil de 5 pour cent. Ce résultat suggère que soit rejetée l'hypothèse nulle, autrement il existe au moins un coefficient différent de zéro.

Deuxièmement, toutes les variables retenues dans l'analyse sont au moins significatives au seuil de 5 pour cent. On remarque que le fait d'être une femme à un impact négatif et significatif sur la probabilité d'occurrence; celle pour un vendeur de pagnes d'accéder au téléphone mobile. Le niveau d'instruction a un effet significatif, plus important que les autres variables, sur la probabilité d’avoir un téléphone portable.

\section{Les contraintes liées à l'accès aux TICs au niveau des vendeurs de pagnes}

En dépit du retard évident du pays dans la diffusion des Technologies de l'Information et de la Communication, certains progrès ont été réalisés, notamment dans la prise de conscience de l'importance de l'infrastructure en télécommunication. Ces efforts demeurent insuffisants et une fraction importante de la population reste privée d'accès aux TICs. Ces problèmes vont freiner considérablement la diffusion de ces technologies au sein des couches sociales défavorisées à court et moyen terme

. Du fait de leur imbrication les uns dans les autres, ils sont présentés sans faire la distinction entre ceux qui sont liés à l'offre ou à la demande. Un de ces problèmes demeure le coût d'acquisition des équipements.

En effet, comme l'a révélé l'enquête, aucun vendeur de pagnes n’a une ligne Internet personnelle à domicile. Cela est en partie dû à l'équipement de base. Les produits informatiques sont à un prix qui exclut cette catégorie de la population. Un ordinateur de bureau neuf est à un prix avoisinant un (1) million de F CFA. En plus, pour un abonnement Internet personnel, il faudrait débourser une somme mensuelle de 49000 F CFA, ce qui est difficilement supportable par des vendeurs de pagnes et particulièrement les détaillants dont les marges de bénéfice sont de $250 \mathrm{~F}$ à $500 \mathrm{~F}$ CFA par pagne.

S'agissant du téléphone mobile, il y a une diffusion considérable au regard du nombre d'abonnés au niveau des trois opérateurs. Une analyse des tarifs permet de mettre en exergue d'autres contraintes, notamment celle qui ne permet pas aux opérateurs de mettre à la disposition des populations des appareils à un prix relativement abordables. 
Les prix de revient des téléphones mobiles de 39000 et 55000 F CFA que proposent les deux opérateurs ne sont toujours pas à la portée de toutes les couches sociales. Ces prix restent en effet élevés au regard du revenu des populations pauvres. L'étude réalisée par le Réseau de Recherche sur les Politiques sociales en Afrique de l'Ouest et du Centre (RPSA/AOC 1998) révèle que la taille moyenne d'un ménage est de 6 personnes à Brazzaville. En outre l'étude estime que le revenu moyen mensuel par tête d'un ménage très pauvre est de $31.284 \mathrm{~F}$ CFA ; celui d'un ménage pauvre est de $70380 \mathrm{~F}$ CFA et celui d'un ménage non pauvre est de 185556 F CFA.

La lecture que l'on peut faire de ces chiffres est que le prix le plus bas d'un appareil en promotion représente 124,66 pour cent du revenu moyen mensuel par tête d'un ménage très pauvre. Il représente 55,41 pour cent dans le cas d'un ménage pauvre et 21,08 pour cent dans le cas d'un ménage non pauvre. Pour un vendeur de pagnes appartenant à la couche sociale des personnes très pauvres et qui épargne $1 / 3$ de son revenu ${ }^{4}$ c'est-à-dire 10428 F CFA, l'achat d'un téléphone mobile au prix de 39000 F CFA correspondrait à environ quatre (4) mois d'épargne. Le même calcul avec la même hypothèse pour les vendeurs de pagnes appartenant à la couche des personnes pauvres correspondrait à environ deux (2) mois d'épargne.

Ces calculs montrent à quel point l'accès au téléphone mobile est difficile pour les couches sociales pauvres. On peut donc avancer sans grand risque de se tromper que l'explosion du téléphone mobile atteint essentiellement les ménages non pauvres qui, selon l'étude citée ci-avant, représente 56,1 pour cent des ménages à Brazzaville. En d'autres termes, le nombre élevé d'abonnés constaté au niveau des différents opérateurs est le fait des ménages non pauvres qui multiplient le nombre d'appareils afin de satisfaire chacun de ses membres.

S'agissant du prix du téléphone et de sa rigidité à la baisse, Monsieur Omère I., consultant auprès des deux opérateurs, donne une explication du phénomène. Pour lui, en effet, il existe une tranche de la population composée des cadres moyens, les commerçants et les étudiants qui ne peuvent acquérir un équipement d'un montant supérieur à 35000 F CFA du fait de leur faible pouvoir d'achat. Mais, suivant les données récentes en sa possession, un équipement bas de gamme, sorti de l'usine coûterait environ 40000 F CFA. Il apparaît de toute évidence qu' avec les charges annexes liées à l'acquisition, qu'un appareil puisse coûter moins de 35000 F CFA. La conséquence logique de cette situation est effectivement la perte d'une partie de la clientèle qui désirerait avoir un appareil.

Par ailleurs, en ce qui concerne les appareils achetés à l'extérieur, $\mathrm{M}$. Omer I. a fait observer que le prix de l'appareil est fixe à la sortie de l'usine. 
Ainsi, l'acquisition d'un appareil à l'extérieur n'est pas non plus inférieure à 40000 F CFA. Cependant en Europe, il existe des contrats d'abonnement qui permettent de mettre l'équipement à la disposition du client à un vil prix tout en contraignant l'abonné à appartenir au réseau pendant une certaine période. Ce type de contrat n'est pas possible dans notre cas pour trois raisons essentielles. D’abord, contraindre un client à rester dans le réseau revient à lui livrer un appareil bloqué sur une bonde passante correspondant à l'opérateur choisi. Or à Brazzaville, il y a une prolifération des maisons spécialisées dans la vente et le déblocage des téléphones mobiles ; cela donne la possibilité au client de changer d'opérateur pour une raison ou une autre. Ensuite un opérateur qui reçoit un nouvel abonné est tout heureux car la valorisation d'une entreprise de téléphone mobile est obtenue au niveau de l'actif et non du passif. Elle est égale au nombre d'abonnés multiplié par la consommation moyenne par abonné. Un abonné de plus est important surtout lorsque la société est côté en bourse. Enfin, au niveau de l'autorité de régulation ${ }^{5}$ on a des textes qui ne sont pas bien précis sur le sujet. Il faudrait donc que les textes soient réécrits et que entre les opérateurs il y ait des accords écrits qui permettent de protéger chacun.

Ce témoignage montre l'existence d'une autre contrainte à savoir : les contrats incomplets. En effet, lorsqu'un opérateur vend un appareil « crypté » à un client, il espère que ce dernier puisse rester pendant un certain temps dans son réseau. Il y a là un accord moral implicite entre les deux. L'opérateur cède l'appareil à un prix partiel et espère récupérer le reliquat avec les consommations du client.

Dans ce contrat liant l'opérateur au client, deux types de coûts liés à l'asymétrie de l'information apparaissent sur le marché. Le premier est évidemment le coût de la vérification. Il provient de la difficulté voire de l'impossibilité pour l'opérateur d'accéder aux perspectives futures des clients, particulièrement lorsque ces derniers sont portés à donner une image plus qu'optimiste d'une collaboration future avec l'opérateur. La sélection adverse se présente donc sur le marché du téléphone mobile parce qu'il existe une asymétrie d'information due à l'incapacité de l'opérateur, qui fait un prêt, d'observer les attributs du client emprunteur et de cerner les contingences sous lesquelles il opère.

Le deuxième coût est celui de la surveillance (monitoring cost). Il se présente lorsque les actions du client emprunteur en référence du contrat moral nécessitent un suivi pour s'assurer qu'aucune faute ne viendrait remettre en cause la promesse de rester dans le réseau pour quelques raisons que se soient. L’aléa de moralité peut provenir sur le marché du téléphone mobile de l'impossibilité ou l'incapacité des opérateurs d'exercer un contrôle sur le 
comportement des clients emprunteurs qui peuvent décrypter l'appareil et changer de réseau.

L'examen des questions d'accès vient de montrer que la mondialisation n'est pas un processus linéaire. Tous les individus ou groupes d'individus ne peuvent bénéficier de ces fruits de la même manière, ne serait-ce à cause des inégalités dans les niveaux de vie, d'organisation, etc. C'est le cas des vendeurs de pagnes qui montre une marginalisation de la femme dans l'accès aux TICs par rapport aux hommes. Aussi, après avoir abordé les problèmes liés à l'accès, il est opportun de s'interroger sur la manière dont ceux qui ont accès aux TICs les utilisent dans le cadre de leur activité. C'est l'objet de la troisième partie de cette étude.

\section{Utilisation des TICs dans le commerce des pagnes}

La tendance au niveau du commerce international est à la constitution des réseaux télématiques (Lediberder 1983, Brousseau 1993) qui ont densifié et accéléré les flux d'information d'une manière impressionnante. En effet, être compétitif au niveau des délais et de la qualité est un aspect majeur des stratégies de mondialisation adoptées par les différents acteurs pour faire face à la concurrence. Dans ces conditions, il est impératif de recueillir et de traiter l'information afin d'agir en conséquence rapidement sur le processus de production et de distribution. Ces manœuvres stratégiques sont rendues possibles principalement grâce à l'émergence des technologies de l'information et de la communication ; et à leur application à l'ensemble de la chaîne de production et de distribution. C'est pourquoi, il est indispensable d'évaluer le degré d'utilisation des TICs dans le commerce des pagnes à Brazzaville. Cette tâche préliminaire conduira ensuite à l'analyse des canaux de transmission de l'information dans cette activité.

\section{Degré d'utilisation des TICs dans le commerce des pagnes à Brazzaville}

Il est ici important de rappeler que la relation avec les fournisseurs est à appréhender à trois niveaux principalement dans la situation d'oligopole qui caractérise le marché. La première concerne les quelques hommes vendeurs grossistes-détaillants originaires d'Afrique de l'Ouest qui importent les pagnes. On peut dire que leurs fournisseurs se trouvent à l'extérieur. Deuxièmement, les vendeurs de pagnes (détaillants) en majorité femmes dans les marchés de Brazzaville qui s’approvisionnent auprès des vendeurs ressortissant de l'Afrique de l'Ouest (grossistes), qui sont leurs fournisseurs en pagnes en provenance des autres pays. Troisièmement, les pagnes en provenance de Kinshasa ${ }^{6}$ qui sont livrés par les Kinoises ${ }^{7}$ qui sont elles aussi des fournisseurs pour les vendeurs (détaillants) sur les marchés de Brazzaville. 
Les résultats obtenus par l'enquête mettant en relief le sexe des vendeurs, la possession ou non du téléphone mobile et l'utilisation du téléphone mobile pour la recherche des fournisseurs montrent que les contacts avec les fournisseurs se font moins par téléphone que les vendeurs en possèdent ou non. C'est dans le groupe des femmes que les pourcentages sont les plus insignifiants. Ils ne dépassent pas 30 pour cent. A contrario chez les hommes, les pourcentages sont de l'ordre de 50 pour cent. Ce premier constat donne l'image de la domination des hommes sur le marché des pagnes. L'analyse du rôle des technologies de l'information et de la communication dans la relation avec la clientèle témoigne d'avantage de cette assertion.

En effet, l'investigation sur deux aspects particulièrement importants de la relation avec la clientèle que sont l'annonce auprès de la clientèle de l'arrivage des pagnes et le recouvrement des fonds, éclaire davantage sur les inégalités entre les hommes et les femmes dans l'utilisation des TICs et partant le contrôle de l'activité par les hommes. À cet effet, les résultats de l'enquête suggèrent que les vendeurs de pagnes utilisent très peu le téléphone mobile dans leurs relations avec la clientèle. Les pourcentages des vendeurs qui utilisent cet instrument pour annoncer l'arrivage de nouveaux motifs et de ceux qui l'utilisent aux fins de recouvrement auprès de la clientèle ne dépassent pas 30 pour cent. Cette barre n'est pas également franchie lorsque l'on considère le groupe des femmes.

En ce qui concerne des femmes vendeuses qui ne disposent pas de téléphone mobile, les pourcentages sont encore plus faibles. Ils ne dépassent pas 10 pour cent dans les deux cas envisagés ici dans la relation avec la clientèle. Néanmoins, l'existence des pourcentages supérieurs à 0 pour cent témoigne de la présence sur ce marché de quelques femmes, qui n’ont pas de téléphone, mais se servent des cabines téléphoniques dans leur relation avec la clientèle.

Au niveau des hommes, les pourcentages des vendeurs de pagnes qui se servent du téléphone mobile dans la relation avec la clientèle sont plus élevés que ceux enregistrés chez les femmes. Toutefois, ces pourcentages ne dépassent pas 50 pour cent. Et, contrairement aux femmes, l'écart entre ceux qui disposent d'un téléphone mobile et ceux qui n'en disposent pas n'est pas grand. Ces résultats se comprennent dans la mesure où les hommes vendeurs de pagnes sont en majorité des grossistes qui expriment des besoins de communication avec les détaillants se trouvant dans les différents marchés de Brazzaville d'une part et l'organisation de l'activité des grossistes ${ }^{8}$ qui exige des contacts permanents avec l'extérieur d'autre part. 


\section{La circulation de l'information dans le commerce des pagnes à Brazzaville}

La manière dont l'information circule est indispensable pour une compréhension bien meilleure de l'utilisation des TICs dans le commerce des pagnes. La première tâche à accomplir dans ce cadre est de dénombrer tous les agents qui interviennent dans cette activité. À cet égard, on dénombre trois types d'agents sur le marché intérieur : les grossistes-détaillants, les détaillants et les consommateurs. Il n'y a pas de producteurs sur place et donc les pagnes vendus sur les marchés à Brazzaville proviennent exclusivement de l'extérieur. Deux agents principalement sont distingués au plan extérieur : les fournisseurs du Congo-Kinshasa et les autres fournisseurs extérieurs. La nécessité de distinguer le marché de Kinshasa des autres marchés extérieurs vient du fait que cette ville, de par sa proximité, constitue un débouché important et en même temps une source d'approvisionnement. En effet, les commerçantes kinoises, via les circuits informels, vendent aux détaillants de Brazzaville les pagnes « Sotexki » qui sont produits à Kinshasa. En repartant, elles achètent auprès des grossistes-détaillants de Brazzaville les pagnes en provenance de la Chine, de Côte d'Ivoire, etc. Ceci s'explique par le fait que l'importation des pagnes est interdite en RDC pour protéger l'industrie nationale. L'analyse est faite en distinguant la circulation verticale et la circulation horizontale de l'information.

En ce qui concerne la circulation de l'information entre les fournisseurs de Kinshasa et les détaillants de Brazzaville, il faut dire que les vendeurs se déplacent le plus souvent et s'informent donc sur place. Le téléphone est utilisé dans des situations exceptionnelles; notamment lorsqu'il y a des commandes des dessins (ou des motifs) non disponibles sur le marché. L'extrait de l'interview de Madame Jeannette Y., vendeuse au marché de PotoPoto donne un éclairage de l'utilisation du téléphone dans ce type de relation.

\section{À propos des relations avec les commerçantes de Kinshasa}

« Il existe en faite trois possibilités. La première est que les commerçantes viennent nous proposer les pagnes et nous les achetons sur place. La seconde possibilité est que nous leurs confions les pagnes chinois (sultana) qu'elles vendent à Kinshasa et en retour elles nous ramènent les pagnes "sotexki" pour la même valeur. Bien entendu, ce partenariat existe avec des personnes avec lesquelles nous avons l'habitude de collaborer depuis des années. La troisième possibilité est que les commerçantes kinoises ne trouvent pas de preneurs au marché, elles vendent les pagnes aux grossistes et nous allons à notre tour nous approvisionner auprès de ces derniers ». 


\section{Dans le cas d'une commande importante}

« Si je ne peux pas satisfaire toute la commande, j’associe les autres. Vous savez ? Nous sommes ici en coopérative et je dirais que nous formons une famille, donc la collaboration est très bonne entre nous. Dans le cas où nous ne réussirions pas à satisfaire la commande, nous recourons aux grossistes ouest-africains. Enfin, si la commande n'est toujours pas satisfaite, je peux téléphoner à une partenaire installé à Kinshasa. Je lui communique alors toutes les instructions (les dessins, le nombre et la date de livraison) pour qu'elle m'aide à répondre aux besoins exprimés par mes clients. À l'acheteur, je lui exige de verser une caution qui dépend de l'importance de la commande. Il peut arriver que ma partenaire de Kinshasa ne saisisse pas l'instruction liée à la qualité du pagne ; à ce moment je lui fais un courrier dans lequel je joins un échantillon du tissu ».

En ce qui concerne la circulation de l'information entre les grossistes et les détaillants, deux cas peuvent être relevés. Les deux cas sont essentiellement basés sur la distance qui sépare les grossistes des détaillants, de sorte que l'utilisation plus ou moins fréquente des TICs dépend de celle-ci. À cet égard, il y a lieu de révéler que la vingtaine de grossistes dénombrés est regroupée au marché de Poto-Poto.

Dans ce marché par exemple, lors de l'arrivage de pagnes avec de nouveaux motifs, les grossistes recrutent une personne qui se charge de ventiler l'information auprès des vendeurs de pagnes dans ce marché (premier cas). Pour les marchés éloignés, il peut passer un coup de fil à une personne qui se charge de la diffusion de l'information ${ }^{9}$. A ce niveau aussi intervient la circulation horizontale de l'information. L'information reçue par un vendeur ou un groupe de vendeurs est diffusé par vague aux autres vendeurs non encore informés. Deux interviews réalisées auprès de deux détaillantes permettent d'étayer ces propos.

\section{Sur l'utilisation du téléphone mobile}

« Madame Jeannette Y.: La plupart des grossistes auprès desquels nous nous approvisionnons ont des démarcheurs. Ce sont donc ces derniers qui viennent sur place nous informer de l'arrivage de nouveaux pagnes. Ensuite, nous nous déportons sur les lieux ».

« Julienne N.: Nous l’utilisons certes (parlant du téléphone mobile), mais vous savez que nous ne gagnons pas " grand chose ». Le fait d'acheter la marchandise sur place ne nous permet pas de faire des gains importants. Bien qu'étant regroupé en coopérative, nous n'avons pas quelqu'un de sûr qui voyage pour aller acheter les pagnes à l'extérieur afin que nous gagnions un peu plus. Par exemple, la 
marge de bénéfice sur une pièce est de 250 F CFA à 500 F CFA pour les pagnes “sotexki”, “chinoi”, wax et autres ».

La circulation de l'information entre les détaillants et les consommateurs, d'une part, entre les grossistes et les détaillants, d'autre part, est marquée par une utilisation au moins du téléphone, bien qu’à petite échelle. En effet, c'est à ce niveau qu'une petite partie de vendeurs acceptent d'utiliser régulièrement le téléphone pour annoncer aux consommateurs réguliers, généralement des travailleurs, l'arrivage de pagnes avec de nouveaux motifs. Cependant, il faut révéler que ces consommateurs réguliers dépassent rarement le nombre de trois. Pour la plupart, les clients sont attendus sur place, c'està-dire au marché. Cela limite l'utilisation des TICs dans cette branche de la circulation de l'information.

La circulation de l'information entre les fournisseurs de Kinshasa et les vendeurs en détail et entre les fournisseurs de Kinshasa et les clients (nonvendeurs de pagnes) via le téléphone portable, est circonstancielle. En effet, l'analyse des différentes interviews réalisées auprès des détaillants a révélé que ces dernières ont des numéros de téléphone qui leurs permettent de joindre leurs fournisseurs de Kinshasa en cas de besoins. Il va s'en dire que la circulation de l'information entre les vendeuses kinoises et les détaillants se fait dans une certaine mesure par le téléphone mobile. Quant à la circulation de l'information entre les fournisseurs de Kinshasa et les clients (consommateurs), le mode le plus utilisé est celui de bouche à oreille. Le téléphone mobile n'a qu'un rang secondaire.

C'est plutôt dans la circulation de l'information entre grossistes et partenaires extérieurs (les grossistes-détaillants de Kinshasa, et d'autres pays) que l'utilisation du téléphone portable est régulière. L’interview accordée par Monsieur Camara I. donne ici un éclairage sur la circulation de l’information à ce niveau.

« Le téléphone portable nous aide beaucoup. Par exemple si quelqu'un a besoin de beaucoup de pagnes, je lui fais un prix de gros et je passe la commande à mon fournisseur. Je lui communique la quantité de pagnes demandée par téléphone. On fait la commande sans se déplacer. J'ai des clients. Ils sont nombreux; surtout à Kinshasa. Ils s’informent régulièrement sur l'arrivage des marchandises. Si elles ne sont pas encore arrivées, alors je les informe sur la date où elles arriveront. Tu sais grâce au téléphone, j’ai des contacts réguliers en Europe, en Asie ...on a des contacts ».

« Avec les détaillants qui vendent dans les marchés, j’ai des contacts par téléphone. Ils demandent ce que j’ai comme nouveauté, les dates d’arrivage. Quelqu'un m’a demandé 10 pièces ce matin au téléphone. Avant il lui fallait prendre un bus pour venir ici. Le téléphone mobile est très important ». 
Au regard de la circulation de l'information sur le marché, il est indispensable de tirer les enseignements relatifs au rôle du téléphone portable sur le fonctionnement du marché. Pour ce faire, il faut d'abord relever le fait que les intérêts des différents agents présents sur le marché divergent. Il y a les grossistes qui veulent maximiser leurs profits en écoulant tous leurs stocks de marchandises quelle que soit sa qualité. Les détaillants ont également un objectif de profit, mais pour être sûr de l'atteindre, il faut qu'ils aient une marchandise de qualité afin de la vendre facilement aux consommateurs. Enfin les consommateurs qui ont pour objectif d'optimiser leur utilité en achetant un produit de qualité et surtout, c'est une spécificité du consommateur brazzavillois, que ce produit ne soit pas répandu de manière à créer la différence. En tenant compte des différents comportements, le téléphone portable va jouer un rôle plus ou moins de « commissaire priseur » sur le marché de walras.

En effet, comme le commissaire priseur, le téléphone permet la fluidité de l'information, et donc permet à ce que les agents sur le marché soit parfaitement informés sur les différentes opportunités. Dans une telle perspective, le grossiste peut informer rapidement ses clients potentiels (détaillants) sur les différents marchés et par vague l'information se propage à tous les autres vendeurs presque simultanément. Mais l'existence des associations de vendeurs sur les différents marchés biaise le fonctionnement du marché en concurrence parfaite. Ces associations fonctionnent comme des cartels dans la mesure où, en amont les vendeurs font leurs commandes ensemble pour bénéficier des prix de gros et donc réduire le coût d'achat des marchandises et une fois sur le marché, ils déterminent le prix minimum de vente des pagnes. Seulement, la fragilité de ces cartels vient du fait que l'information reçue sur le marché est également exploitée par les vendeurs n'appartenant pas aux cartels, c'està-dire aux associations de vendeurs de pagnes. Ces vendeurs, qui visent aussi la maximisation du profit, vont très vite constituer en amont un groupe informel, de circonstance pour bénéficier des prix de gros.

L'avantage vient ici du fait que ces groupes informels de 5 personnes en moyenne peuvent se constituer en une journée et se présenter chez les grossistes en premier et bénéficier d'un choix plus large. Cette rapidité de réaction n'est pas possible au niveau des associations qui sont constituées de plus de 50 personnes. Il faut déléguer une poignée de personnes chez le grossiste pour collecter les motifs et les ramener aux autres, ensuite chacun des vendeurs doit faire son choix et faire enregistrer sa commande. Enfin, la commission fait le point et procède à l'achat de la marchandise. Ce processus peut prendre 3 à 5 jours avec le risque que certains motifs ne soient plus disponibles. C'est le premier inconvénient lié au fonctionnement des associations de vendeurs. 
Le deuxième inconvénient vient du fait que sur le marché, les membres de l'association ont un prix minimum pour chacun des pagnes en tenant compte de la qualité. La fixation d'un prix seuil rompt également avec le principe du fonctionnement libre du marché car en concurrence parfaite celui-ci est sensé s'ajuster en fonction des quantités offertes et demandées sur le marché. Cette situation de prix seuil favorise encore les vendeurs non membres des associations. Ces derniers comme des passagers clandestins peuvent se permettre de baisser leurs prix en dessous du prix seuil fixé par les associations. Cette situation n'est pas exceptionnelle car les grossistesdétaillants pratiquent aussi des prix inférieurs à ceux des différentes associations. Cela suppose une réduction de la marge bénéficiaire, mais l'élévation des recettes et de la fréquence de rotation de l'activité contribue bien à optimiser le profit. Cette situation à la longue pourra contribuer à l'éclatement des associations si elles ne changent pas leur mode de fonctionnement.

Ce premier niveau d'analyse montre que si le téléphone portable permet d'assurer une information permanente et parfaite entre les grossistes et les détaillants, se sont plutôt les comportements opportunistes des agents qui contribuent le plus à introduire un biais dans le fonctionnement d'un marché supposé en concurrence parfaite c'est-à-dire avec des produits homogènes et des acheteurs uniformes, l'atomicité des acheteurs et des vendeurs, une information parfaite pour tous les agents et une entrée et une sortie libre. A contrario, la circulation de l'information entre les détaillants et les consommateurs par le téléphone portable ne permet pas non plus le fonctionnement normal du marché en concurrence parfaite.

En effet, le premier fait à relever est que les consommateurs s'informent en général sur place, en faisant la ronde des détaillants et des grossistesdétaillants. Dans ces conditions, la circulation de l'information via le téléphone portable ne concerne que les consommateurs réguliers ayant même créer des affinités avec les vendeurs. Mais lorsque les consommateurs sont informés de l'arrivage de nouveaux motifs, la tendance n'est pas à la diffusion de ceux-ci ne serait-ce pour éviter que le pagne acheté soit répandu dans la ville. Il y a donc rétention de l'information et il n'est pas possible dans ces conditions d'avoir une information parfaite sur le marché. Ici encore le téléphone portable ne permet pas, à cause du comportement des agents, de jouer le rôle de commissaire priseur, indispensable au fonctionnement du marché en concurrence parfaite Il ne permet pas aux commerçants de faire des gains de productivité.

L'analyse de la circulation de l'information a permis une bonne compréhension d'un faible niveau d'utilisation du téléphone mobile et de l'Internet. Au-delà il fait prendre conscience de la marginalisation des femmes qui sont les plus nombreuses, mais pourtant, elles n'ont pas le contrôle de 
l'activité. Enfin l'analyse de la circulation de l'information a montré que c'est dans les relations entre les fournisseurs et les vendeurs et entre les fournisseurs et les partenaires extérieurs que les TICs sont le plus utilisées. Il s'agit de la relation entre les marchés extérieurs (Kinshasa compris) et les grossistes et de la relation entre les détaillants et les grossistes ainsi que les fournisseurs en provenance de Kinshasa. Les relations entre les clients (consommateurs) et les autres agents, notamment les grossistes et les fournisseurs kinois sont marquées par l'utilisation des moyens de communication traditionnelle : de bouche à oreille. Cette conclusion remet en selle le débat sur la productivité induite par les TIC.

\section{Incidence des TICs sur le commerce des pagnes à Brazzaville}

Après avoir abordé les problèmes d'accès et d'utilisation des TICs dans le commerce des pagnes, on serait tenté de penser que cette dernière partie aurait perdu toute sa raison être. En effet, le faible niveau d'utilisation de ces innovations dans l'activité de vente des pagnes laisse envisager un impact général peu significatif. Pour autant une telle conclusion reste sommaire; elle ne permet pas de se rendre compte de la moindre perception du gain, soit-til minime, par les vendeurs de pagnes (premier point). De même cette conclusion ne fait de distinction entre les incidences dans la relation avec la clientèle d'une part, et avec les fournisseurs, d'autre part. Car, le fait d'être peu ou beaucoup utilisés ne justifie toujours pas l'importance de l'impact des outils de communication sur l'activité.

\section{La perception du gain dû aux TICs par les vendeurs de pagnes}

En dépit du faible niveau d'accès et donc d'utilisation des TICs, l'objectif ici est de savoir si les gains inhérents à cette utilisation - aussi minimale soit-elle - sont perceptibles par les vendeurs de pagnes. À cet égard il a été demandé aux vendeurs de pagnes de donner leurs points de vue sur l'incidence des TICs sur leurs recettes. Les résultats obtenus suggèrent que moins de 35 pour cent des vendeurs de pagnes admettent que les TICs ont contribué à l'augmentation des ventes. Ce pourcentage concerne les vendeurs qui ont un téléphone portable. Pour ceux qui n'en disposent pas, ce pourcentage ne dépasse pas 5 pour cent. Il a été constaté, en outre que, l'écart entre les hommes et les femmes disposant des téléphones mobiles n'est pas grand, mais les deux proportions n'atteignent pas 40 pour cent. A contrario, lorsqu'on considère les groupes de vendeurs n'ayant pas de téléphones mobiles, les proportions baissent. Cette dernière est plus flagrante chez les femmes (2,74 pour cent). Ces résultats sont triviaux au regard des développements de la deuxième partie de cette étude. Une fois encore, les extraits d'entretiens avec les vendeurs de pagnes sont mieux placés pour expliquer ces résultats. 


\section{Pensez-vous que le téléphone mobile a une incidence significative dans votre activité ?}

« Jeannette Y.(détaillante): Oh! Pas du tout. Il me permet certes de communiquer quelques fois avec les commerçantes de Kinshasa lorsque j'ai une commande importante, mais pour autant je ne peux pas dire qu'il a un impact significatif sur mon activité ».

« M. Camara I. (grossiste) : Le téléphone nous fait gagner dans l’activité. Par exemple, avec les partenaires de Kinshasa je peux m'informer au téléphone si la marchandise est disponible...si les pagnes sont disponibles à l'usine. Avant on payait un billet pour aller à Kinshasa, mais maintenant avec 300 F CFA je peux être informé. Il fallait même un visa de $25000 \mathrm{~F}$ CFA pour aller à Kinshasa...là avec une minute de communication et on est satisfait. Le téléphone mobile nous a permis également d'élargir notre activité. En dehors des facilités, je vends à côté des pagnes maintenant beaucoup d'autres choses telles que les téléphones, et leurs accessoires ».

L’attitude de resserve adoptée par la détaillante traduit celle de l'ensemble rencontré dans les marchés. Les détaillants étant les plus nombreux, il advient que le pourcentage des vendeurs qui pensent que le téléphone mobile a un effet peu significatif sur leur activité est ipso-facto moins important. Mais la différence entre la détaillante et le grossiste traduit aussi une différence dans le niveau d'utilisation. La deuxième partie de cette étude a montré que les grossistes utilisent le téléphone mobile dans leur activité plus que les détaillants.

En ce qui concerne l'Internet, une telle analyse s'avère difficile dans la mesure où la quasi-totalité des vendeurs de pagnes ne l'utilise pas dans l'exercice de leur activité. Beaucoup ne savent pas l'utiliser et ont à peine entendu parler de cette innovation comme le témoigne ces interviews de madame Jeannette Y. et monsieur Camara I.

\section{Q: Avez-vous déjà entendu parler de l'Internet ?}

« Jeannette Y.: J'en ai entendu parler pour la première fois quand Koffi Olomidé (chanteur populaire de la RDC) a sorti un album dans lequel il en a fait état. Je ne sais pas exactement ce que c'est ».

«Camara I. : l'Internet est important, je ne l'ai pas encore appris, mais j'entends parler. Comme l'activité est en baisse en ce moment je vais l'apprendre. L'Internet comme le téléphone sont très utile dans le commerce. Mais, entre le téléphone et l'Internet, le téléphone est le premier». 


\section{Analyse économétrique de l'incidence des TICs sur le commerce des pagnes}

Afin de procéder à une vérification empirique de l'incidence des TICs sur le commerce des pagnes, un modèle économétrique est estimé. L’objectif ici est de vérifier si l'utilisation des TICs dans cette activité a un impact positif sur les ventes journalières. En effet, l'amélioration et la rapidité dans le traitement de l'information rendue possible par les TICs devraient se traduire par de meilleures ventes. Dès lors ces ventes journalières sont considérées être dépendantes du nombre d'heures de travail par jour, de l'expérience du vendeur, pris en compte ici par le nombre d'années dans l'exercice de l'activité et de l'usage des TICs aux fins d'exercices de cette activité. Les recettes journalières (variable endogène) sont supposées reliées aux variables exogènes par une fonction log-linéaire.

Les variables exogènes comprennent: le nombre d'heures de travail moyen journalier que consacre le vendeur à son activité, la variable TIC qui prend la valeur 1 lorsque le vendeur utilise au moins l'une des TICs pour contacter ses clients et 0 lorsque ce n'est pas le cas. La variable représente quant à elle le contact avec les fournisseurs. Comme la précédente variable, elle prend la valeur 1 lorsque le vendeur utilise l'une des TICs pour contacter ses fournisseurs et 0 lorsque ce n'est pas le cas.

L'estimation de cette équation s'est faite à partir des données d'enquêtes auprès des vendeurs. Le problème statistique qui se pose lors de l'estimation de ce type de modèle, celui de l'hétéroscédasticité (c'est-à-dire celui de l'inconstance de la variance du terme de l'erreur). Ce dernier a été résolu en appliquant systématiquement la correction de White lors de l'estimation.

Il sied d'abord de signaler que deux indicateurs renseignent sur la qualité globale de la régression : la statistique " $\mathrm{R}^{2}$ " et la statistique de Fisher. La statique " $\mathrm{R} 2$ " qui indique le degré d'adéquation du modèle au données est à un niveau qui peut être considéré comme étant bas. Ce résultat n'est pas surprenant car les recettes journalières dépendent aussi des variables structurelles telles que la chute du pouvoir d'achat des ménages à la suite de la dévaluation, de la réduction des salaires et de l'appauvrissement des ménages après les guerres de 1997 et de 1998 qui ne sont pas pris en compte dans la spécification de ce modèle. Au niveau de la statistique de Ficher, la probabilité pour que les coefficients des variables explicatives soient nuls est extrêmement faible. Cela amène à conclure que le modèle est globalement satisfaisant au seuil de 5 pour cent.

L'analyse des coefficients suggère que l'expérience et le nombre d'heures de travail soient significatifs au seuil de 5 pour cent. En travaillant une heure de plus par jour, le vendeur augmente ses recettes journalières de 0,88 pour 
cent. De même en augmentant le nombre d'années d'exercice de l'activité d'une année (accroissement de l'expérience), les recettes journalières vont augmenter de 0,12 pour cent.

S'agissant des deux variables retenues pour capturer l'effet des TICs sur le commerce des pagnes, il y a lieu de relever le caractère contrasté de l'incidence des deux. La variable « contact avec les fournisseurs » a un coefficient positif. Cela suggère que l'utilisation des TICs par les vendeurs dans la relation avec leurs fournisseurs ait un effet positif sur les recettes journalières. Ce résultat n'est pas surprenant dans la mesure où les TICs permettent aux vendeurs de retrouver très vite des motifs recherchés par les clients. Ils peuvent alors s'approvisionner rapidement et faire face, dans les meilleurs délais, aux exigences des clients.

La variable « contact avec la clientèle » quant à elle présente un coefficient avec un signe négatif. Ce résultat suggère que les relations qu'établissent les vendeurs de pagnes avec la clientèle par les TICs ne permettent pas de justifier le niveau des recettes journalières de ces derniers. Cela se traduit donc par ce signe négatif. En outre il y a lieu de révéler que ce coefficient n'est pas significatif au seuil de 5 pour cent, ce qui amène à dire que l'utilisation des TICs dans les relations avec la clientèle a un effet marginal sur les ventes journalières.

Les résultats obtenus sur l'incidence des TICs dans le commerce des pagnes reflètent le degré d'utilisation dans les relations vendeurs - fournisseurs d'une part et vendeurs - consommateurs d'autre part. Les TICs sont assez utilisées dans le premier cas alors que dans le deuxième c'est une communication de bouche à oreille qui domine, d'où les résultats obtenus.

\section{Conclusions et implications de politique}

Au terme de cette étude, les conclusions suivantes peuvent être dégagées à trois niveaux : l'accès, l'utilisation, l'incidence.

- L’accès : il ressort que le degré d'accès reste bas pour l'ensemble des vendeurs de pagnes, d'une part, et même en considérant les deux groupes : les hommes et les femmes, d'autre part. Malgré ces faibles taux de pénétration, la différence entre les hommes et les femmes est très nette. Le pourcentage d'hommes disposant d'un téléphone est vraiment supérieur à celui des femmes. Il en est de même pour l'Internet.

- L'utilisation : la première leçon que l'on peut tirer de toutes les analyses est que les TICs sont plus utilisées entre les détaillants et les grossistes et surtout entre les grossistes et les fournisseurs extérieurs. Ainsi, la circulation de l'information entre les clients et les autres agents sur le marché se fait très rarement avec les TICs. Ici également, l’utilisation des TICs 
pour faire circuler l'information entre les différents intervenants sur ce marché, a montré des différences importantes entre les hommes (respectivement les femmes) disposant d'un téléphone mobile d'une part et entre les hommes et les femmes dans chacun des cas évoqués ci-avant d'autre part.

- L'incidence des TICs sur le commerce des pagnes : le premier enseignement à tirer de cette étude est que les proportions des vendeurs de pagnes qui perçoivent les gains dus aux TICs sont faibles pour l'ensemble et pour chaque groupe. C'est toujours dans le groupe des hommes que les résultats sont importants par rapport aux femmes. Le deuxième enseignement vient de l'analyse économétrique. Cette dernière a révélé que c'est l'utilisation des TICs dans les relations avec les fournisseurs qui a un impact significatif sur les recettes journalières. Le niveau d'utilisation des TICs dans les relations avec les clients n'est pas suffisant pour expliquer les recettes journalières dans cette activité.

Les conclusions tirées à ces trois niveaux d'analyse confirment bien l'hypothèse de travail sur les différences d'accès entre les hommes (les femmes) d'une part et entre les hommes et les femmes d'autre part. Il en est de même de l'hypothèse sur les effets limités des TICs qui est vérifiée au regard des conclusions liées à l'incidence des TICs sur le commerce des pagnes.

Le problème primordial qu'il convient de relever ici, plus que celui de l'utilisation et l'incidence, est celui de l'accès à ces TICs pour les vendeurs de pagnes en général et en particulier les femmes vendeuses, qui sont largement majoritaires dans l'activité, mais nullement la maîtrise de cette dernière. En effet, l'accès aux TICs dans le contexte de la mondialisation s'impose pour ces femmes, comme un impératif majeur. C'est une question de survie dans cette activité fortement tournée vers l'extérieur, autrement cette activité pourtant rentable, deviendrait pour elles une simple activité de production et de reproduction de la force de travail. Ainsi, tendre et s'engager pour relever ce défi, est la seule voie pour les femmes vendeuses de pagnes de sortir de la marginalisation et de l'exclusion afin de prétendre jouer les premiers rôles dans cette activité. Car, ce n'est pas la technologie en soie qui exclue, mais au contraire, ce sont les modalités mises en œuvre et l'attitude ou l'état d'esprit qu'on observe qui entraîne l'exclusion ou la marginalisation (Babassana 2000).

Au regard de ce qui précède, les orientations de politique suivantes, portant sur les voies et les moyens de diffusion de ces technologies et de leur maîtrise par les populations en général et les vendeurs de pagnes en particulier, peuvent 
être formulées. Un rôle moteur de l’État, qui doit définir une stratégie et des politiques appropriées. Plus précisément l'État et l'opérateur historique doivent jouer un rôle de premier plan dans l'orientation générale, l'implantation des infrastructures et de gros équipements.

La deuxième condition est constituée par la mise en œuvre des modalités ou des dispositifs qui permettent au grand public d'y accéder. Ces dispositifs peuvent passer par : les télécentres, des cybercafés, l'introduction des TICs notamment l'Internet dans le système éducatif. Il s'agit pour les différents intervenants de définir une politique de formation multidimensionnelle, notamment en ce qui concerne l'apprentissage de l'informatique à l'école primaire et secondaire, à l'enseignement supérieur, ainsi que dans le cadre de la formation continue à tous les niveaux.

Pour les vendeuses de pagnes, il s'agit précisément, d'une part, de les amener à prendre conscience de ce que la maîtrise de leur activité va dépendre de plus en plus de la maîtrise de ces nouveaux outils de communication. D'autre part, il serait important de concilier le programme de formation avec les possibilités de disponibilité des femmes vendeuses de pagnes. Pour le téléphone mobile, les ONG pourront par exemple jouer un rôle d'agent dans le cadre de l'établissement d'une relation d'agence entre l'opérateur, les vendeurs de pagnes et l'ONG afin de faciliter l'accès à cette catégorie de consommateurs potentiels. Quant à l'Internet, une question reste ouverte : quel est le prix supportable par les vendeurs de pagnes et qui assure la rentabilité de l'activité du formateur d'autant plus que le consentement à payer reste très faible?

\section{Notes}

1. Le nombre de points de communication ici renvoie au nombre de cybercafés et de cabines téléphoniques.

2. Par cette expression nous voulons dire que le téléphone portable est une technologie plus facilement accessible aux hommes qu'aux femmes.

3. Le prix de $500 \mathrm{~F}$ CFA est réservé aux personnes qui savent naviguer. Elles peuvent prendre 30 minutes de navigation et ouvrir une boîte électronique. Le prix de $750 \mathrm{~F}$ CFA est quant à lui réservé aux personnes ne sachant pas naviguer. EIles paient de ce fait un supplément de $250 \mathrm{~F}$ CFA pour l'assistance qu'elles bénéficient lors de la navigation.

4. Une telle hypothèse sous-entend que l'individu devrait réduire sa consommation alimentaire et surtout ne pas tomber malade, ce qui est difficilement réalisable d'autant plus qu'une bonne santé dépend en partie d'une bonne alimentation.

5. Direction générale de l'Administration centrale des Postes et Télécommunications (DGACPT). 
6. Les femmes via le commerce transfrontalier vendent des pagnes « SOTEXKI » et en repartant, elles achètent les pagnes chinois, et autres pour les revendre à Kinshasa.

7. On désigne ainsi les habitantes de Kinshasa.

8. Les investigations menées à ce sujet ont révélé que les grossistes ont bien des contacts avec des fournisseurs extérieurs qui leurs communiquent les prix des pagnes régulièrement. Les grossistes se regroupent et recensent les besoins de chacun d'eux et délèguent un pour l'achat. Le transport de la marchandise et son dédouanement fait l'objet d'une cotisation chacun au prorata de son volume de commande. Le billet quant à lui fait l'objet des cotisations à égal montant entre les grossistes.

9. Il convient de relever, là aussi, que l'information qui arrive est globale. On annonce seulement l'arrivage des pagnes, mais on ne mentionne rien sur la qualité de ces derniers. Les vendeurs doivent ensuite se rendre sur place pour s’enquérir de la qualité des pagnes arrivés.

\section{Références}

Adera, Edith, 2001, Experiences in Gender and ICTs for Development : An IDRC perspective, communication présentée au séminaire national sur l'information relativeaux femmes, National Women's Education Center, Japon, 16 mars.

Akerlof, G., 1970, « The market for lemons : quality uncertainty and the market mechanism », The Quarterly Journal of Economics, 84 : 488-500.

Babassana, Hilaire, 2000, «Accélération des nouvelles technologies de l'information et de la communication et émergence et expansion d'une nouvelle économie », communication présentée au Forum de la semaine Congolaise des nouvelles technologies de l'information et de la communication, Brazzaville du 13 au 17 juin, CREP.

Bisilliat, Jeanne, 2000, « Luttes féministes et développement : une perspective historique », Cahier genre et développement : le genre un outil nécessaire, Paris : L'Harmattant, AFED, Genève : EFI : 19-29.

Brousseau, E., 1993, « L’économie des contrats, technologies de l'information et coordination interentreprises », Économie en liberté, Paris : PUF : 137 - 177.

Cahuc, P., 1993, La nouvelle microéconomie, Paris : La Découverte.

FNUAP, 2000, Vivre ensemble, dans des mondes séparés : hommes et femmes à une époque de changements, Prographics, Inc., États-Unies.

Heckman, J, 1979, « Sample selection bias as a spécification error », Econometrica, vol. 52, n³ : 542 - 562.

Initiatives Genre et Développement, 2002, « L’approche genre », http :// www.igedmadagascar.org/approche.

Jeune Afrique intelligent, 2000, « La guerre du téléphone, Congo (Brazzaville) », $\mathrm{n}^{\circ}$ 2076-2077.

Khayat, Mustapha, 1994, « L’échange de données informatisées dans les activités d'exportation des pays du sud : les passages portuaire ", Revue Tiers Monde, Paris : PUF : 375-390. 
Kodjo, Edem, 1996, «Les enjeux politiques et économiques des nouvelles technologies de l'information pour l'Afrique », Afrique 2000, revue trimestriel n²5, octobre-novembre-décembre : 7-13.

Lediberder, A., 1983, La production des réseaux de télécommunication, Paris : Economica.

Locoh, Thérèse, 1996, « Changement des rôles masculins et féminins dans la crise : la révolution silencieuse ", in Coussy, Jean et Vallin, Jacques (dir), Crise et population en Afrique, Paris : CEPED : 445-469.

Marot, Christelle, 2001, « L'actualité des secteurs », Marché tropicaux, novembre.

Mike, Jensen, 2001, « Afriboîtes, Télécentre et Cybercafés : Les TIC en Afrique », Coopération Sud, $\mathrm{n}^{\circ} 1: 112-127$.

PNUD, 2001, « Mettre les nouvelles technologies au service du développement humain », Rapport mondial sur le Développement humain, New York: PNUD.

Raimberg, P., 1995, « Asymétrie d'information, théorie de l'agence et gestion de l'entreprise », Encyclopédie de gestion, Paris : Economica : 181-191.

Ricardo-Gomez, Juliana Martinez et Reilly, Kathérine, 2001, "Au-delà de la connectivité : l'expérience de l'Amérique Latine et des Caraïbes », Coopération Sud, $\mathrm{n}^{\circ} 1: 128-142$.

RSPA/ AOC, 1998, Alternative stratégique de la lutte contre la pauvreté au Congo, Rapport final de recherche, URSPA, RSPA/ AOC, CRDI, CRVZ, Brazzaville.

Varian, Hal R., 1997, Introduction à la microéconomie, Paris : De Boeck-Université, Nouveau Horizons. 
